\title{
SOME RECENT ADVANCES ON THE MODELING OF THE HYSTERETIC BEHAVIOR OF RATE-INDEPENDENT PASSIVE ENERGY DISSIPATION DEVICES
}

\author{
Nicolò Vaiana, Ciro Napolitano, and Luciano Rosati \\ Department of Structures for Engineering and Architecture, University of Naples Federico II \\ via Claudio, 21, 80124 Naples, Italy \\ e-mail: nicolo.vaiana@unina.it
}

\begin{abstract}
Structures with passive energy dissipation systems are characterized by a complex dynamic behavior due to the hysteretic nature governing the response of the adopted devices. Mathematically speaking, the hysteretic models currently available to simulate the behavior of rate-independent devices, such as metallic and friction dampers, are much more complicated than those developed for rate-dependent devices, such as viscous fluid and viscoelastic solid dampers. To allow for a straightforward, accurate and efficient modeling of rate-independent energy dissipation devices in practice, we introduce a novel phenomenological model suitable for both metallic and friction dampers. Such a model offers the advantage of requiring an easy calibration procedure since it is based on a set of only four parameters having a clear mechanical significance. The proposed model is employed to simulate the actual behavior of some metallic dampers, denominated Shear Links, that have been experimentally tested at the University of Naples Federico II. In addition, numerical simulations are performed to show the capability of such devices in protecting structures from earthquake excitations.
\end{abstract}

Keywords: Energy Dissipation Device, Hysteretic Behavior, Nonlinear Dynamic Analysis, Phenomenological Model, Shear Link. 


\section{INTRODUCTION}

Passive energy dissipation represents one of the most effective techniques for the seismic protection of buildings and bridges [1, 2, 3, 4]. This technique requires the use of special devices, called dampers, that are characterized by a high energy dissipation capacity and are generally incorporated in the external frames of a structure thus dissipating energy throughout its entire height [5].

Dampers typically exhibit a complex hysteretic behavior. In particular, such a behavior is referred to as rate-independent if the device restoring force depends on the device displacement, whereas it is defined rate-dependent when the device restoring force is a function of the device velocity. Clearly, there may exist some dampers showing both types of behavior at the same time [6].

Examples of rate-independent passive energy dissipation devices, commonly used for seismic protection of structures, are metallic dampers and friction dampers, whereas examples of rate-dependent devices are viscous fluid dampers and viscoelastic solid dampers.

Several hysteretic models have been proposed in the literature for simulating the complex behavior occurring in dampers [7]. Typically, rate-independent hysteretic models are much more complex than the rate-dependent ones from a mathematical point of view [8].

Among existing rate-independent models, the Bouc-Wen model $[9,10]$ and its modified versions [7] seem to be the most suitable ones since they allow for an accurate prediction of the hysteretic response of both metallic and friction dampers by using a relatively small number of parameters. Unfortunately, these models require parameters with no clear mechanical meaning thus involving the use of complex and time-consuming identification procedures. In addition, due to their differential nature, they are not computationally efficient since they require the numerical solution of a first-order nonlinear ordinary differential equation for the evaluation of the hysteretic variable at each time step of a nonlinear dynamic analysis.

To foster the use of accurate and efficient rate-independent models in practice, this paper proposes a novel phenomenological model able to predict the hysteretic behavior generally displayed by metallic and friction dampers. Compared to the celebrated Bouc-Wen model or its modified versions, the proposed model, belonging to a more general class formulated in $[11,12,13]$, is based on a set of only four parameters having a clear mechanical significance. For such a reason, it needs a straightforward calibration procedure and, consequently, it may be easily adopted in practice.

The paper is organized into three parts. In the first part (Sections 2 and 3), we first derive the nonlinear equilibrium equations of a typical building frame equipped with energy dissipation devices and subjected to earthquake excitation. Subsequently, we briefly describe, for the reader's convenience, the three main categories of dampers with particular emphasis on the description of their hysteretic behavior.

In the second part (Section 4), we present a preliminary formulation of the proposed hysteretic model suitable for rate-independent dampers, such as metallic and friction devices, and then we illustrate the influence of the four parameters on the hysteresis loop size and/or shape.

Finally, in the third part (Section 5), we adopt the proposed hysteretic model to simulate the experimental behavior of a particular class of metallic dampers, denominated Shear Links, and we perform several nonlinear dynamic analyses on a building frame equipped with such devices to show their capability in reducing both floor relative displacements and absolute accelerations. 


\section{NONLINEAR EQUILIBRIUM EQUATIONS}

The response of a Multi-Degree-Of-Freedom (MDOF) system is generally governed by the following system of coupled second-order nonlinear Ordinary Differential Equations (ODEs):

$$
\mathbf{M} \ddot{\mathbf{u}}(t)+\mathbf{C} \dot{\mathbf{u}}(t)+\mathbf{K u}(t)+\mathbf{f}_{n}(t)=\mathbf{p}(t),
$$

in which $\mathbf{u}(t), \dot{\mathbf{u}}(t)$, and $\ddot{\mathbf{u}}(t)$ are the generalized displacement, velocity, and acceleration vectors, respectively, whereas $\mathbf{M}, \mathbf{C}$, and $\mathbf{K}$ represent the generalized constant mass, damping, and elastic stiffness matrices, respectively; in addition, $\mathbf{f}_{n}(t)$ is the generalized nonlinear force vector, which may have rate-independent and/or rate-dependent hysteretic nature, whereas $\mathbf{p}(t)$ represents the generalized external force vector depending on time $t$.

In this work, we specialize such nonlinear equilibrium equations to the structural model of a typical building frame equipped with energy dissipation devices, each mounted in combination with two diagonal braces. In particular, we introduce a 3-story two-dimensional (2D) structural model whose geometry is defined in a global, right-handed Cartesian coordinate system $(O, Y$, $Z$ ), as shown in Figure 1.

For simplicity, we assume that: a) frame elements, such as beams, columns, and braces, deform within their linear elastic range during the earthquake excitation, whereas the hysteretic devices exhibit nonlinear behavior; b) columns are axially inextensible; c) beams and braces are axially inextensible and flexurally rigid. We observe that these assumptions, typically introduced in the literature [14], can be removed without any influence on the proposed results.

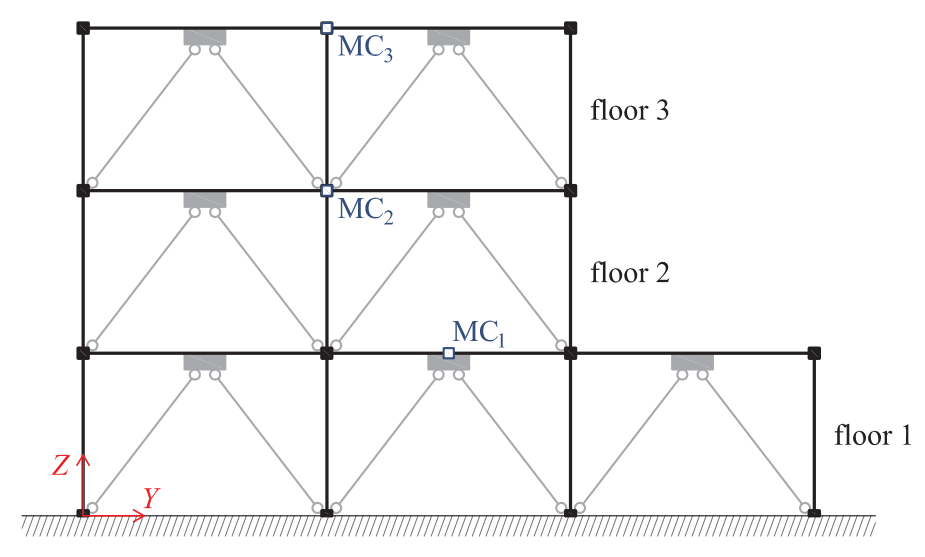

Figure 1: 2D structural model of a typical building frame with added dampers.

The above-described structural idealization allows us to write the displacement, velocity, and acceleration vectors of the 2D structural model, relative to the ground, as follows:

$$
\mathbf{u}=\left\{\begin{array}{l}
u^{(1)} \\
u^{(2)} \\
u^{(3)}
\end{array}\right\}, \quad \dot{\mathbf{u}}=\left\{\begin{array}{l}
\dot{u}^{(1)} \\
\dot{u}^{(2)} \\
\dot{u}^{(3)}
\end{array}\right\}, \quad \ddot{\mathbf{u}}=\left\{\begin{array}{c}
\ddot{u}^{(1)} \\
\ddot{u}^{(2)} \\
\ddot{u}^{(3)}
\end{array}\right\},
$$

where $u^{(i)}, \dot{u}^{(i)}, \ddot{u}^{(i)}$ represent, respectively, the displacement, velocity, and acceleration along the $Y$-axis of the $i$-th floor. 
On account of that, the constant mass, damping, and elastic stiffness matrices become:

$$
\begin{array}{rlr}
\mathbf{M}=\left[\begin{array}{rrr}
M^{(1)} & 0 & 0 \\
0 & M^{(2)} & 0 \\
0 & 0 & M^{(3)}
\end{array}\right], \quad \mathbf{C}=\left[\begin{array}{rrr}
C^{(1)}+C^{(2)} & -C^{(2)} & 0 \\
-C^{(2)} & C^{(2)}+C^{(3)} & -C^{(3)} \\
0 & -C^{(3)} & C^{(3)}
\end{array}\right], \\
\mathbf{K}=\left[\begin{array}{rrr}
K^{(1)}+K^{(2)} & -K^{(2)} & 0 \\
-K^{(2)} & K^{(2)}+K^{(3)} & -K^{(3)} \\
0 & -K^{(3)} & K^{(3)}
\end{array}\right],
\end{array}
$$

where $M^{(i)}$ is the $i$-th floor mass, $C^{(i)}$ is the $i$-th floor damping coefficient, whereas $K^{(i)}$ is the $i$-th floor elastic stiffness, obtained by summing the elastic stiffnesses of the $i$-th floor columns.

Finally, the nonlinear force vector and the external force vector are given by:

$$
\mathbf{f}_{n}=\left\{\begin{array}{l}
f_{n}^{(1)} \\
f_{n}^{(2)} \\
f_{n}^{(3)}
\end{array}\right\}, \quad \mathbf{p}=-\left[\begin{array}{rrr}
M^{(1)} & 0 & 0 \\
0 & M^{(2)} & 0 \\
0 & 0 & M^{(3)}
\end{array}\right]\left\{\begin{array}{l}
1 \\
1 \\
1
\end{array}\right\} \ddot{u}_{g},
$$

where $f_{n}^{(i)}$ represents the nonlinear force of the $i$-th floor, whereas $\ddot{u}_{g}$ is the ground acceleration applied along the $Y$-axis. In particular, if the vector $\mathbf{f}_{n}$ is a function of the displacement vector $\mathbf{u}$, its three components are computed as:

$$
\begin{aligned}
f_{n}^{(1)} & =\sum_{j=1}^{3} f_{j}^{(1)}\left(u^{(1)}\right)-\sum_{j=1}^{2} f_{j}^{(2)}\left(u^{(2)}-u^{(1)}\right), \\
f_{n}^{(2)} & =\sum_{j=1}^{2} f_{j}^{(2)}\left(u^{(2)}-u^{(1)}\right)-\sum_{j=1}^{2} f_{j}^{(3)}\left(u^{(3)}-u^{(2)}\right), \\
f_{n}^{(3)} & =\sum_{j=1}^{2} f_{j}^{(3)}\left(u^{(3)}-u^{(2)}\right)
\end{aligned}
$$

where $f_{j}^{(i)}$ is the rate-independent hysteretic force exhibited by the $j$-th damper of the $i$-th floor.

Conversely, if the vector $\mathbf{f}_{n}$ is a function of the velocity vector $\dot{\mathbf{u}}$, the displacement $u^{(i)}$, appearing in Equation 5 , has to be substituted with the velocity $\dot{u}^{(i)}$; in such a case, $f_{j}^{(i)}$ would represent the rate-dependent hysteretic force displayed by the $j$-th damper of the $i$-th floor.

\section{ENERGY DISSIPATION DEVICES}

Several types of passive energy dissipation devices have been adopted worldwide to protect structures from earthquake excitations. According to the nature of their hysteretic behavior, such devices may be classified into three categories [7]:

1. rate-independent hysteretic devices: dampers whose restoring force does not depend on the rate of variation of the displacement (i.e., velocity) but only upon the displacement amplitude and the sign of the velocity (i.e., direction of the motion). Some examples are represented by metallic dampers and friction dampers.

2. rate-dependent hysteretic devices: dampers whose restoring force depends, partially or totally, on the rate of variation of the displacement (i.e., velocity). Examples of such devices are viscous fluid dampers and viscoelastic solid dampers. 


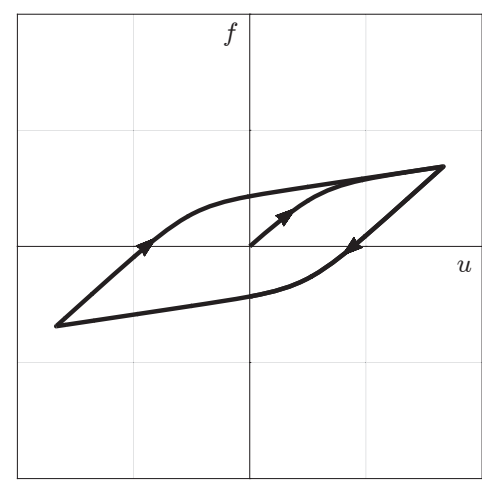

(a)

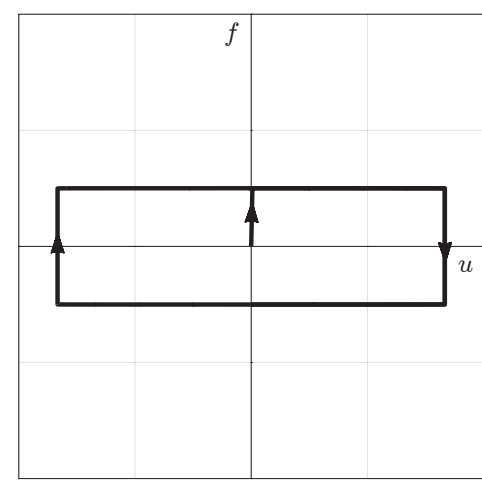

(b)

Figure 2: Typical hysteresis loop of (a) metallic and (b) friction dampers.

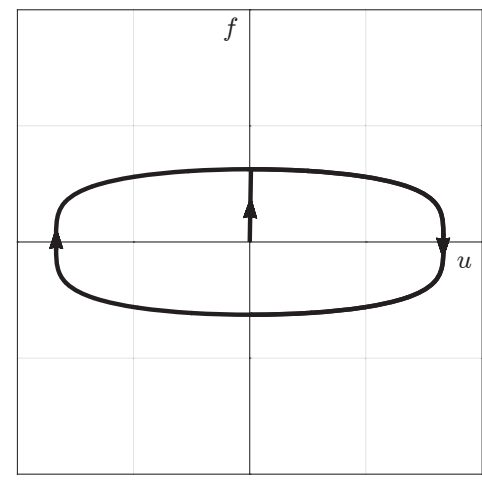

(a)

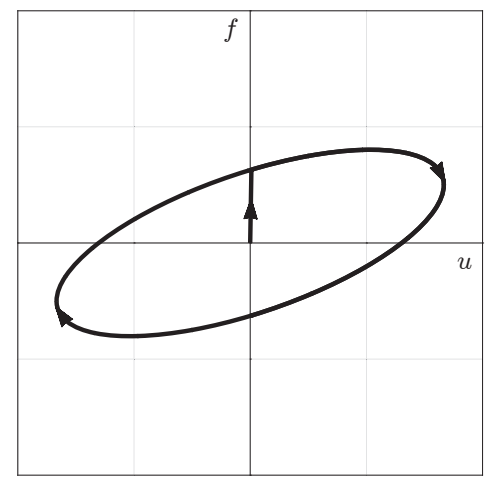

(b)

Figure 3: Typical hysteresis loop of (a) viscous fluid and (b) viscoelastic solid dampers.

3. further devices: dampers which cannot be classified by one of the above-described basic types. Some examples are friction-spring devices with re-centering capability and fluid restoring force-damping devices.

Figure 2a (b) illustrates the typical force-displacement hysteresis loop of a metallic (friction) damper, whereas Figure 3a (b) shows the hysteresis loop which generally characterizes a viscous fluid (viscoelastic solid) damper.

Clearly, the hysteresis phenomena observed in these devices are induced by different types of mechanisms. As a matter of fact, in metallic dampers such a nonlinear behavior is associated with yielding of mild steel, whereas in friction dampers it is due to the sliding friction across the interface between two solid bodies.

Furthermore, in viscous fluid dampers the energy dissipation is due to the friction between fluid particles and the piston head, whereas in viscoelastic solid dampers it is related to the deformation of solid elastomeric pads.

\section{PROPOSED HYSTERETIC MODEL}

In this Section, we focus on the modeling of rate-independent hysteretic devices which, differently from the rate-dependent ones, require much more complex hysteretic models.

In particular, we first summarize the formulation of a novel uniaxial phenomenological 
model belonging to a more general class introduced by Vaiana et al. [11, 12, 13, 15]; subsequently, we illustrate the influence of the four model parameters on the size and/or shape of the restoring force-displacement hysteresis loops.

\subsection{Model Formulation}

In the Proposed Hysteretic Model (PHM), $u(f)$ represents the damper axial/transverse displacement (restoring force), whereas $\dot{u}$ is the damper axial/transverse velocity.

The restoring force, during the generic loading case $(\dot{u}>0)$, is evaluated as:

$$
f\left(u, u_{j}^{+}\right)= \begin{cases}c^{+}\left(u, u_{j}^{+}\right) & u \in\left[u_{j}^{+}-2 u_{0}, u_{j}^{+}\right] \\ c_{u}(u) & u \in\left[u_{j}^{+}, \infty\right),\end{cases}
$$

whereas, during the generic unloading case $(\dot{u}<0)$, it is computed as:

$$
f\left(u, u_{j}^{-}\right)= \begin{cases}c^{-}\left(u, u_{j}^{-}\right) & u \in\left[u_{j}^{-}, u_{j}^{-}+2 u_{0}\right] \\ c_{l}(u) & u \in\left(-\infty, u_{j}^{-}\right] .\end{cases}
$$

In Equations 6 and 7, $c^{+}$and $c^{-}$represent, respectively, the generic loading and unloading curves:

$$
\begin{aligned}
& c^{+}\left(u, u_{j}^{+}\right)=k_{b} u+f_{0}+\frac{k_{a}-k_{b}}{c_{1}}\left[+u-u_{j}^{+}-\alpha_{1} \log \left(c_{1}+e^{\frac{+u-u_{j}^{+}+2 u_{0}-\alpha_{2}}{\alpha_{1}}}\right)+c_{2}\right], \\
& c^{-}\left(u, u_{j}^{-}\right)=k_{b} u-f_{0}-\frac{k_{a}-k_{b}}{c_{1}}\left[-u+u_{j}^{-}-\alpha_{1} \log \left(c_{1}+e^{\frac{-u+u_{j}^{-}+2 u_{0}-\alpha_{2}}{\alpha_{1}}}\right)+c_{2}\right],
\end{aligned}
$$

whereas $c_{u}$ and $c_{l}$ are, respectively, the upper and lower limiting curves:

$$
\begin{aligned}
& c_{u}(u)=k_{b} u+f_{0}, \\
& c_{l}(u)=k_{b} u-f_{0} .
\end{aligned}
$$

The internal variable $u_{j}^{+}\left(u_{j}^{-}\right)$, that represents the displacement value where the generic loading (unloading) curve intersects the upper (lower) limiting curve, is given by:

$$
\begin{aligned}
& u_{j}^{+}=+\alpha_{1} \log \left\{c _ { 1 } ^ { - 1 } \left[e^{+\frac{u_{P}+c_{2}-c_{1}\left(k_{a}-k_{b}\right)^{-1}\left(f_{P}-k_{b} u_{P}-f_{0}\right)}{\alpha_{1}}}-e^{\left.\left.\frac{+u_{P}+2 u_{0}-\alpha_{2}}{\alpha_{1}}\right]\right\},}\right.\right. \\
& u_{j}^{-}=-\alpha_{1} \log \left\{c_{1}^{-1}\left[e^{-\frac{u_{P}-c_{2}-c_{1}\left(k_{a}-k_{b}\right)^{-1}\left(f_{P}-k_{b} u_{P}+f_{0}\right)}{\alpha_{1}}}-e^{\frac{-u_{P}+2 u_{0}-\alpha_{2}}{\alpha_{1}}}\right]\right\},
\end{aligned}
$$

in which $u_{P}$ and $f_{P}$ are the coordinates of a generic point $P$ belonging to $c^{+}$or $c^{-}$.

As regards the internal parameters $f_{0}$ and $u_{0}$, they are evaluated as:

$$
\begin{gathered}
f_{0}=\frac{k_{a}-k_{b}}{2 c_{1}}\left(2 u_{0}-c_{2}\right), \\
u_{0}=\frac{1}{2}\left[\alpha_{2}+\alpha_{1} \log \left(\frac{k_{a}-k_{b}}{\delta_{k}}-c_{1}\right)\right],
\end{gathered}
$$

where $\delta_{k}$ is a numerical parameter set equal to $10^{-20}$, whereas $k_{a}, k_{b}, \alpha_{1}$ and $\alpha_{2}$ represent the four model parameters to be calibrated from experimental or numerical data, with $k_{a}>k_{b}$, $k_{a}>0, \alpha_{1}>0, \alpha_{2}>0$. Finally, $c_{1}$ and $c_{2}$ are two constants whose expressions, depending on the four model parameters and omitted for brevity, will be illustrated in detail in a future work. 


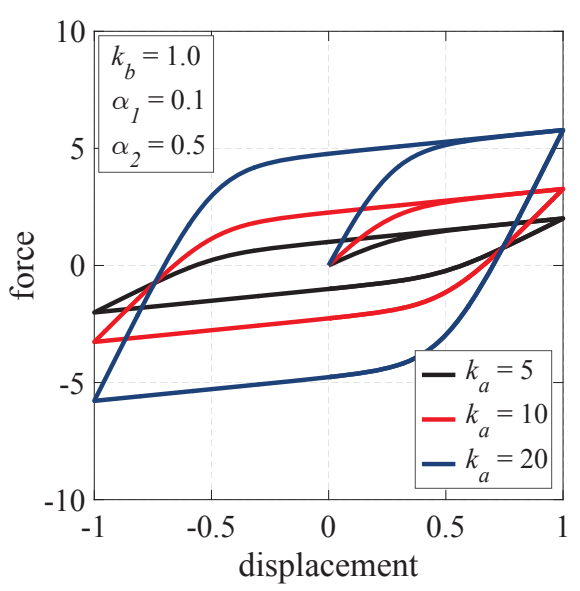

(a)

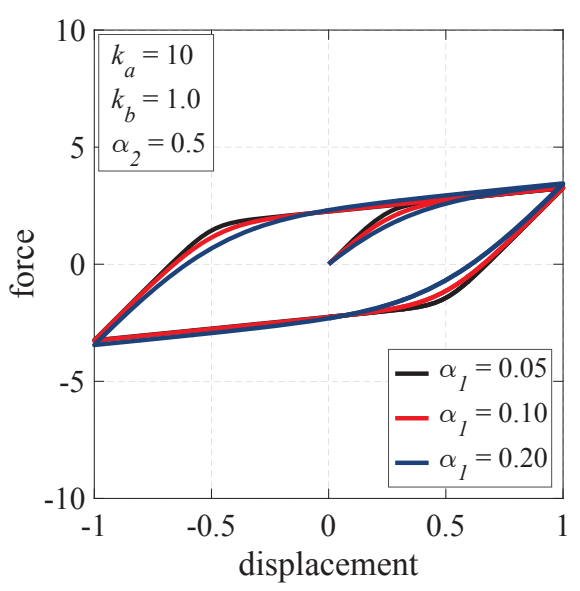

(c)

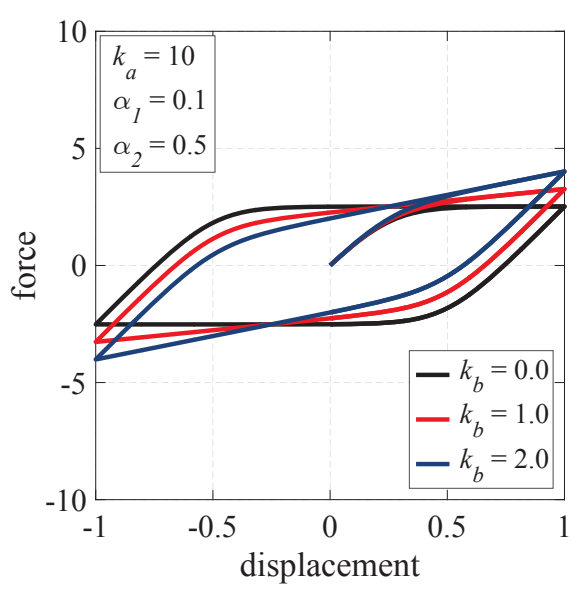

(b)

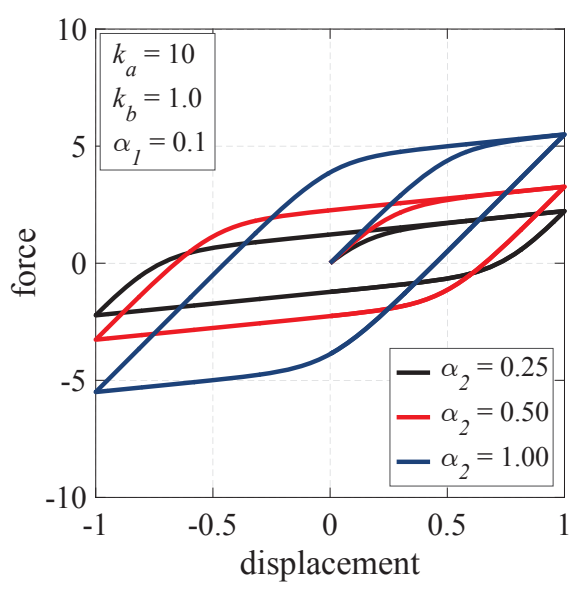

(d)

Figure 4: Influence of the PHM parameters variation on the hysteresis loop size and/or shape: (a) $k_{a}$, (b) $k_{b}$, (c) $\alpha_{1}$, and (d) $\alpha_{2}$.

\subsection{Parameter Sensitivity Analysis}

Figure 4 shows how the size and (or) the shape of hysteresis loops, obtained by imposing a full sinusoidal cycle of displacement having unit amplitude and frequency, change(s) due to the variation of each PHM parameter, namely $k_{a}, k_{b}, \alpha_{1}$ and $\alpha_{2}$.

\section{NUMERICAL EXPERIMENTS}

This Section illustrates the results of several nonlinear time history analyses performed on a building frame equipped with energy dissipation devices and subjected to earthquake excitation. In order to demonstrate the effectiveness of the adopted passive control system and its capability to significantly decrease both floor relative displacements and absolute accelerations, the numerical results are compared with those obtained by analyzing the same building frame, having linear elastic behavior, without using the hysteretic devices.

To carry out the nonlinear dynamic analyses, the proposed hysteretic model, described in Section 4, is employed to simulate the behavior of each damper adopted in the passive control system. Furthermore, an accurate and computationally efficient explicit time integration method, described in [8], is adopted to numerically integrate the system of nonlinear equilib- 


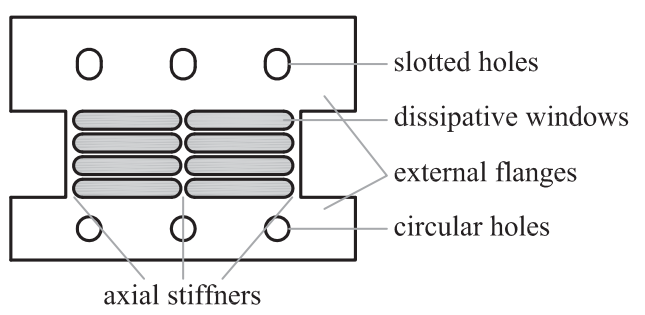

(a)

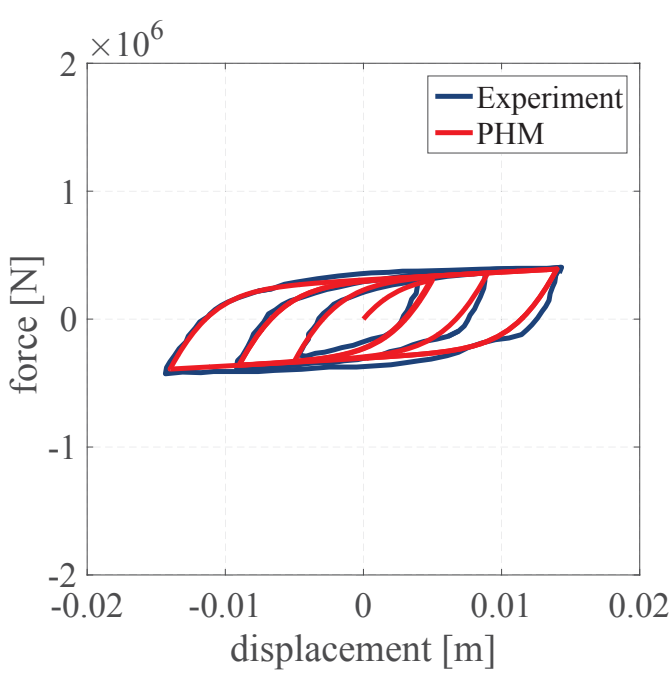

(b)

Figure 5: Shear Link: (a) typical lateral view and (b) comparison between experimental and analytical hysteresis loops of device $S L \_30 \_3$.

rium equations. Due to its explicit nature, such a method offers the advantage of performing nonlinear dynamic analyses without adopting iterative procedures.

Both the hysteretic model and the numerical method have been implemented in the computer program MATLAB.

\subsection{Structure Properties}

Figure 1 shows the 2D structural model of the building frame selected to perform the numerical experiments. In particular, it is a three-story, three-bay reinforced concrete structure whose beams (columns) have a length of $6.0 \mathrm{~m}(3.2 \mathrm{~m})$ and are characterized by a rectangular section with size $0.3 \mathrm{~m} \times 0.4 \mathrm{~m}(0.5 \mathrm{~m} \times 0.5 \mathrm{~m})$.

The masses of the first, second, and third floor are equal to $85200 \mathrm{Ns}^{2} \mathrm{~m}^{-1}, 57400 \mathrm{Ns}^{2} \mathrm{~m}^{-1}$, and $42600 \mathrm{Ns}^{2} \mathrm{~m}^{-1}$, respectively, and are assumed to be lumped in the respective floor mass center, namely $M C_{1}, M C_{2}$, and $M C_{3}$.

As a result of the assumptions made in Section 2, the total number of Degrees-Of-Freedom (DOFs) is equal to 3, whereas the three natural periods, evaluated without taking into account dampers and steel braces, are $T_{1}=0.2220 \mathrm{~s}, T_{2}=0.0916 \mathrm{~s}$, and $T_{3}=0.0630 \mathrm{~s}$. As typically done with reinforced concrete structures, it is assumed that each mode has a damping ratio of $5 \%$ [14].

The passive control system consists of 7 dampers which are mounted between the steel braces and the upper beam, as illustrated in Figure 1. The adopted devices, denominated Shear Links, are metallic dampers, having rate-independent hysteretic behavior, that are manufactured from a I-shaped laminated steel plate [16,17]. As shown in Figure 5(a), this type of device is typically made up of a) vertical stiffeners, that ensure the device stability, b) external flanges, that allow for the device connection to structural elements, and c) dissipative windows, that are capable of dissipating energy. Bolted connections are employed to facilitate the installation process; in particular, slotted holes are used for the upper connection in order to avoid axial forces on the device.

The Shear Links employed in this paper have been experimentally tested by Nuzzo et al. [18] 


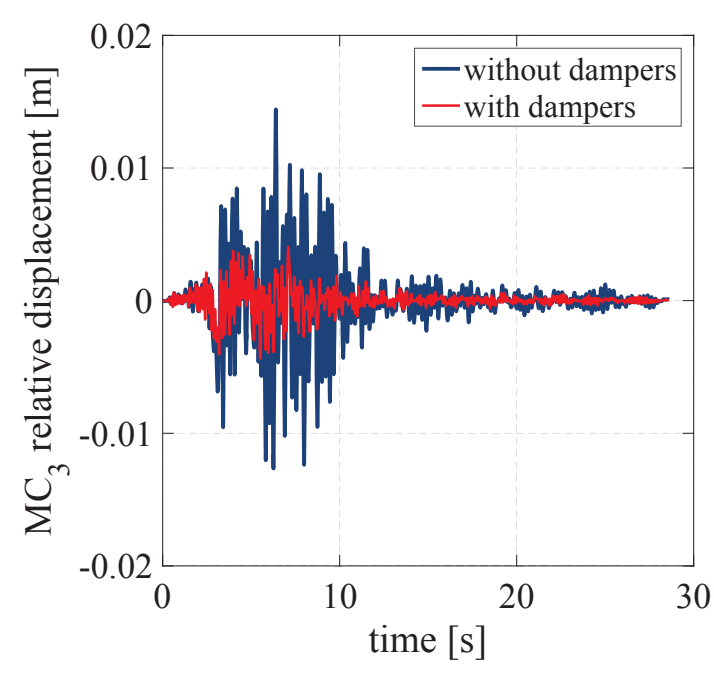

(a)

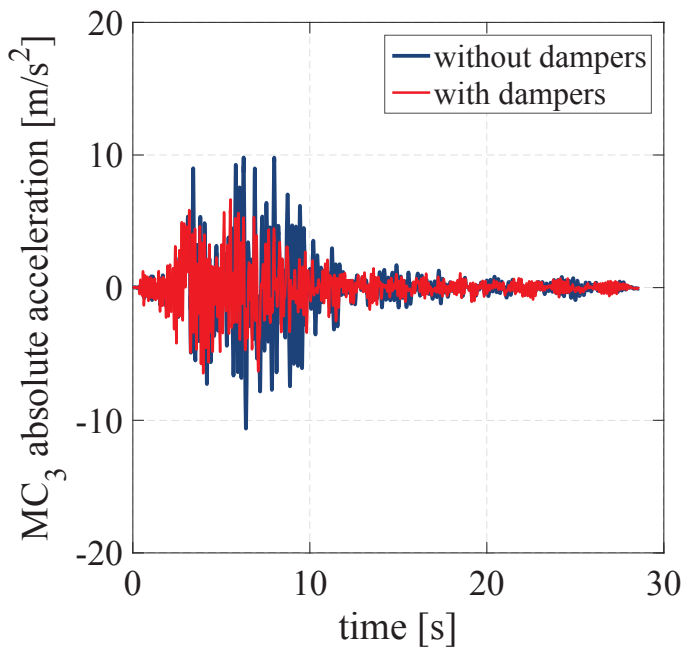

(b)

Figure 6: Time histories of the $M C_{3}$ (a) relative displacement and (b) absolute acceleration obtained with (red line) and without (blue line) metallic dampers.

at the laboratory of Department of Structures for Engineering and Architecture of the University of Naples Federico II (Italy). In particular, the device adopted in the numerical simulation, denominated $S L \_30 \_3$, exhibits the restoring force-displacement hysteresis loops of Figure 5(b) when subjected to a displacement time history having increasing amplitude [18].

\subsection{Applied earthquake excitation}

The analyses are performed by imposing, along the $Y$-axis, the component $\mathrm{SN}$ of the 1994 Northridge motion, and by adopting a ground acceleration record time step of $0.005 \mathrm{~s}$.

\subsection{Hysteretic model parameters}

The complex behavior of each Shear Link is simulated with the novel phenomenological model, presented in Section 4, whose 4 parameters have been calibrated on the basis of the experimental hysteresis loops obtained by Nuzzo et al. [18]. In particular, the values identified for such parameters are: $k_{a}=2 \times 10^{8} \mathrm{~N} / \mathrm{m}, k_{b}=6 \times 10^{6} \mathrm{~N} / \mathrm{m}$, and $\alpha_{1}=\alpha_{2}=0.002 \mathrm{~m}$.

Figure 5(b) compares the experimental hysteresis loops of the device $S L \_30 \_3$ with those predicted by means of the proposed hysteretic model thus demonstrating a satisfactory agreement between them.

\subsection{Numerical results}

Figure 6(a) illustrates the time histories of the $M C_{3}$ displacement, relative to the ground, obtained with (red line) and without (blue line) metallic dampers; similarly, Figure 6(b) shows the time histories of the $M C_{3}$ absolute acceleration obtained with (red line) and without (blue line) Shear Links. Looking at such comparisons, it is evident that the adopted passive protection system is capable of limiting potential damages induced by seismic events since it allows for the reduction of both floor relative displacements and absolute accelerations. 


\section{CONCLUSIONS}

We have presented a preliminary formulation of a novel uniaxial phenomenological model that is capable of simulating the complex behavior of both metallic and friction dampers by employing four parameters having a clear mechanical significance.

The proposed model has been calibrated on the basis of some experimental hysteresis loops obtained by Nuzzo et al. [18] during an experimental campaign performed on Shear Link devices. In particular, the accuracy of the model has been verified by comparing the simulated restoring force-displacement hysteresis loops with those obtained from the experimental tests.

In addition, the proposed model has been employed to analyze a building frame equipped with Shear Links. The numerical results obtained by applying an earthquake ground acceleration have demonstrated the capability of the adopted dampers in controlling excessive floor displacements and accelerations.

\section{REFERENCES}

[1] T.T. Soong, B.F. Spencer Jr, Supplemental energy dissipation: State-of-the-art and stateof-the-practice. Engineering structures, 24(3), 243-259, 2002.

[2] A. Calabrese, D. Losanno, M. Spizzuoco, S. Strano, M. Terzo, Recycled Rubber Fiber Reinforced Bearings (RR-FRBs) as base isolators for residential buildings in developing countries: The demonstration building of Pasir Badak, Indonesia. Engineering Structures, 192, 126-144, 2019.

[3] D. Losanno, M. Spizzuoco, G. Serino, Design and retrofit of multistory frames with elastic-deformable viscous damping braces. Journal of Earthquake Engineering, 23(9), 1441-1464, 2019.

[4] D. Losanno, I.E. Madera Sierra, M. Spizzuoco, J. Marulanda, P. Thomson, Experimental performance of unbonded polyester and carbon fiber reinforced elastomeric isolators under bidirectional seismic excitation. Engineering Structures, 209, 110003, 2020.

[5] M.C. Constantinou, M.D. Symans, Seismic response of structures with supplemental damping. The Structural Design of Tall Buildings, 2(2), 77-92, 1993.

[6] M.C. Constantinou, T.T. Soong, G.F. Dargush, Passive energy dissipation systems for structural design and retrofit. MCEER Monograph No. 1, State University of New York, Buffalo, NY, USA, 1998.

[7] M.D. Symans, F.A. Charney, A.S. Whittaker, M.C. Constantinou, C.A. Kircher, M.W. Johnson, R.J. McNamara, Energy dissipation systems for seismic applications: Current practice and recent developments. Journal of Structural Engineering, 134(1), 3-21, 2008.

[8] N. Vaiana, S. Sessa, F. Marmo, L. Rosati, Nonlinear dynamic analysis of hysteretic mechanical systems by combining a novel rate-independent model and an explicit time integration method. Nonlinear Dynamics, 98(4), 2879-2901, 2019.

[9] R. Bouc, Modele mathematique d'hysteresis. Acustica, 24(1), 16-25, 1971.

[10] Y.K. Wen, Method for random vibration of hysteretic systems. Journal of the Engineering Mechanics Division, 102(2), 249-263, 1976. 
[11] N. Vaiana, S. Sessa, F. Marmo, L. Rosati, A class of uniaxial phenomenological models for simulating hysteretic phenomena in rate-independent mechanical systems and materials. Nonlinear Dynamics, 93(3), 1647-1669, 2018.

[12] N. Vaiana, S. Sessa, L. Rosati, A generalized class of uniaxial rate-independent models for simulating asymmetric mechanical hysteresis phenomena. Mechanical Systems and Signal Processing, 146, 106984, 2021.

[13] N. Vaiana, D. Losanno, N. Ravichandran, A novel family of multiple springs models suitable for biaxial rate-independent hysteretic behavior. Computers and Structures, 244, 106403, 2021.

[14] A.K. Chopra, Dynamics of structures: Theory and applications to earthquake engineering, 4th Edition. Prentice Hall, 2012.

[15] S. Sessa, N. Vaiana, M. Paradiso, L. Rosati, An inverse identification strategy for the mechanical parameters of a phenomenological hysteretic constitutive model. Mechanical Systems and Signal Processing, 139, 106622, 2020.

[16] I. Nuzzo, D. Losanno, N. Caterino, Seismic design and retrofit of frame structures with hysteretic dampers: A simplified displacement-based procedure. Bulletin of Earthquake Engineering, 17(5), 2787-2819, 2019.

[17] I. Nuzzo, D. Losanno, F. Cilento, N. Caterino, Analytical and numerical modelling of shear-link device for seismic energy dissipation in frame structures. Engineering Structures, 214, 110630, 2020.

[18] I. Nuzzo, D. Losanno, N. Caterino, G. Serino, L.M. Bozzo Rotondo, Experimental and analytical characterization of steel shear links for seismic energy dissipation. Engineering Structures, 172, 405-418, 2018. 